\title{
Expert reasoning about independent and dependent variables in thermodynamics
}

\author{
Michael Vignal, ${ }^{1}$ Reese R. Siegel, ${ }^{1}$ Paul J. Emigh, ${ }^{1}$ and Elizabeth Gire ${ }^{1}$ \\ ${ }^{1}$ Department of Physics, Oregon State University, Corvallis, OR 97331
}

\begin{abstract}
Thermodynamic potentials (or free energies) are different energies that can describe the same system using different sets of independent variables. While the flexibility to consider one system in multiple ways is powerful, deciding which variables to treat as independent - and therefore which thermodynamic potential to use - is challenging for students. As part of a larger study investigating student reasoning about independent variables in thermodynamics, we interviewed five thermodynamics experts to learn how they make decisions about independent variables and thermodynamic potentials. This paper outlines the variety of approaches these experts used to determine appropriate independent variables and thermodynamic potentials for given situations.
\end{abstract}

\section{INTRODUCTION}

The concepts of independent and dependent variables are taught in primary school science classes, however little research has been done on students' understanding and use of independent and dependent variables in middle- and upper-division physics courses. In thermal systems, the independent variables inform the selection of a thermodynamic potential (also known as a free energy), which are Legendre transformations of the internal energy. Thermodynamic potentials are powerful tools that provide significant flexibility when considering complicated thermodynamic situations [1]. In a previous study, we asked middle-division physics majors to identify an appropriate thermodynamic potential for a given situation [2]. We found that the students had difficulty determining the independent variables of the system, which hindered their ability to select the appropriate Legendre transformation and thermodynamic potential.

While difficulties with Legendre transformations have been documented [1], our observations of advanced undergraduate physics majors' difficulty with identifying independent variables surprised us. To better understand these observations, we conducted interviews with five experts in thermodynamics to answer the following research questions:

RQIV How do experts determine if a variable is independent in a thermodynamic system?

RQTP How do experts match a thermodynamic potential and a physical system?

\section{METHODS}

We identified five physics faculty members (who will be discussed using pseudonyms) from an R1 research institution who conduct research involving thermodynamics and have, with the exception of Emerson, taught advanced thermodynamics courses. We conducted semistructured interviews with each expert, transcribed audio recordings of the interviews, and coded the transcripts using a new emergent coding scheme [3] that we call Statement Deconstruction (described in Section II B).

\section{A. Protocol}

The interview began with general questions about thermodynamic potentials and Legendre transformations
(IQ1-IQ4). Then we asked the experts to consider specific physical scenarios (IQ5). Most of the interviews were 15-20 minutes, while one (Charlie's) was 45 minutes. The scripted questions were:

IQ1 What is a thermodynamic potential?

IQ2 What role do thermodynamic potentials play in thermodynamics?

IQ3 What are Legendre transformations?

IQ4 What steps do you take to match thermodynamic potentials and physical scenarios?

IQ5 You want to learn as much as possible about 3 scenarios. For each:

- What is your system?

- What experiments could you do?

- What would the independent variables be?

Gas A heavier-than-air gas in an open container.

Ice A sturdy, sealed metal box filled with ice that you put in a heat bath.

Comet A rocky comet that you shine a laser at.

300K (Follow-up) You heat the comet to 300K.

The scenarios in IQ5 were intended to allow the experts to elaborate on the techniques they described in IQ1-IQ4. The Gas scenario closely resembles a question asked of students in previous research [2]. The Ice and Comet scenarios were chosen because they represent (respectively) canonical and exotic thermodynamic situations. The $300 \mathrm{~K}$ follow-up question arose in the first interview and was incorporated into the protocol to be asked in subsequent interviews.

\section{B. Coding}

We transcribed audio recordings of the interviews and coded them using our new Statement Deconstruction emergent-coding structure. From the transcripts, we identified statements the experts made about thermodynamic variables and Legendre transformations. In each statement, we identified a Claim about a variable/potential (e.g., "volume is independent") and a Justification about why the claim is valid (e.g., "because it is held fixed.")

After 4 passes through the transcripts by MV and 1 pass by each RRS and PJE, as well as meetings to refine 
TABLE I. Grouped coding results. Some Justifications fell into multiple categories, so values in the columns do not sum to the totals in the bottom row.

\begin{tabular}{l|cc|cc|c}
\multirow{2}{*}{ Justification Category } & \multicolumn{2}{|c|}{ RQIV } & \multicolumn{2}{|c|}{ RQTP } & \multirow{2}{*}{ Total } \\
& IQ1-4 & IQ5 & IQ1-4 & IQ5 & \\
\hline Control & 3 & 17 & 9 & 6 & 35 \\
Change & 3 & 18 & 12 & 7 & 40 \\
Measurability & 0 & 13 & 1 & 1 & 15 \\
Functional Relationship & 0 & 10 & 0 & 2 & 12 \\
Other & 3 & 5 & 2 & 0 & 10 \\
\hline Total & 7 & 46 & 18 & 11 & 82 \\
\hline
\end{tabular}

definitions and resolve coding discrepancies, we identified and coded 82 statements. The analysis reported here focuses on the Justifications the experts provided for why a variable is or is not independent (RQIV, 53 statements) or how they would select a thermodynamic potential (RQTP, 29 statements).

\section{RESULTS}

We grouped Justifications into five categories (see Table I) with some Justifications existing in multiple categories (see Fig. 1). The numbers in Table I and Fig. 1 provide context for various claims discussed in our analysis, but our analysis is primarily qualitative and not directed by these numbers.

\section{A. Control (35 Statements)}

We coded statements as Control if the participant discussed being able (or explicitly unable) to choose the value of some variable. The literature on Legendre transformations suggests attending to control to identify independent variables and thermodynamic potentials $[1,4]$. Thus, we were not surprised when each of the experts used control to justify at least 3 statements regarding selecting independent variables (RQIV) and thermodynamic potentials (RQTP). For example, Blair strongly associated control with independence:

Blair [Ice]: Since apparently the only thing I can control here is the temperature, you just scan through the whole temperature range.... Temperature is the only control that I have.

$M V:$ Would you consider temperature to be an independent variable in that case?

Blair: Yeah, because that's what you control.

Emerson likewise indicates that controlled variables are independent, and also that dependent variables are not directly controlled:

Emerson: One [independent variable] is the laser power...the dependent variable will be the temperature, so I can increase the laser power and that will increase the temperature accordingly.

The vast majority of expert statements about control indicate that the experts associate controlling a variable with the variable being independent or important for determining an appropriate thermodynamic potential.

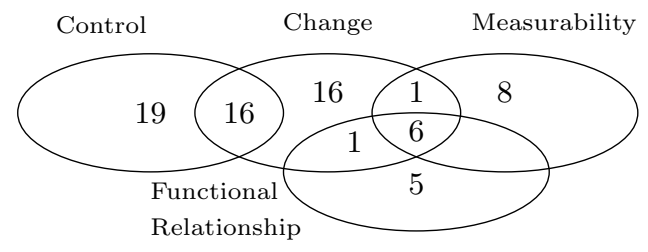

FIG. 1. A Venn diagram showing overlap between Change and the other categories (see Section IV for discussion).

\section{B. Change (40 Statements)}

Statements were coded as Change if the participant discussed the value of a variable over time. All experts except Blair used Change to justify Claims about independent variables (RQIV), while all except Danny used Change to justify statements regarding thermodynamic potentials (RQTP). In half of these 40 statements, experts discussed when the value of a variable changes:

Avery [IQ4]: You have to assess a problem and determine which variables are being changed and then transform to the correct variable... and find the right free energy-Legendre transform.

In the other half of statements in the Change category, experts talk about knowing that a variable is constant, fixed, or has a change of zero:

Emerson [IQ4]: All you have to do is look at the system, whether it is a fixed temperature or a fixed pressure, you know, so you can choose which free energy you're going to use.

Despite the high frequency of the Change code in our analysis of experts' statements, discussion of attending to change to pick a thermodynamic potential is absent from [1] and only briefly mentioned in [4] (and then only with reference to variables being constant). Our experts generally agreed that a variable is independent or important for picking a thermodynamic potential if the change is zero or if the change is not zero but the variable that is changing is being controlled.

Charlie [IQ1]: As you change the volume.... Helmholtz free energy and internal energy give you the answer depending on if you want to keep the entropy fixed versus keeping the temperature fixed.

We address such category overlap further in Section IV.

\section{Measurability (15 Statements)}

The Measurability category contains statements regarding experts being able or deciding to measure the value of a variable. Though every expert discusses Measurability, Measurability is used to justify treating a variable as independent or dependent in different circumstances. For example, Charlie discusses how being able to measure a variable can mean it is independent:

Charlie [300K]: It's reaching a fixed temperature, and temperature is an easy thing to measure, so I should treat that as my [independent] variable. 
At another point in the interview, however, Charlie indicates that measured variables are not independent:

Charlie [Ice]: I want to measure the pressure as a function of temperature, and volume is going to be held fixed, so I'll think of temperature and volume as my two independent variables. I'm going to measure pressure.

\section{Functional Relationship (12 Statements)}

When an expert's Justification was centered on knowing the relationship between two or more variables, we coded it as Functional Relationship. All experts except Blair relate finding Functional Relationships to determining if a variable is independent or not.

Avery [Ice]: I'm just measuring the temperature as a function of time.... So I guess time along my $x$ and temperature along my $y$.

Since the Functional Relationship between time and temperature is unknown in the scenario, Avery indicates that temperature is dependent by placing it on the $y$-axis.

Danny discusses Functional Relationship as important for determining which thermodynamic potential to use:

$\boldsymbol{M V}$ [Gas]: What thermodynamic potentials would you expect to be important?

Danny: You can do either way. So, the easier way to think about this is what is the pressure at different levels and you can work back to the density. You can also partition it in sort of volumes. I don't even know what the easiest way to do this sort of one is, but the volumes, you could sort of divide it and count the particles. I need the equation of state for the particles... Yeah, they both work.

In this case, the equation of state is the Functional Relationship that would allow Danny to pick a thermodynamic potential using either pressure or volume.

\section{E. Other (10 Statements)}

Some of the most interesting expert statements were coded as Other. For example, Danny and Charlie both answer (IQ4) by stating that they attend to independent variables when selecting a thermodynamic potential:

Danny: So you look for independent and dependent variables.

Charlie: What I do is I always think about what I want the independent variables to be.

Further examples of Other Justifications include ideas such as "because it is an ideal gas" and instances when no justification is given.

\section{DISCUSSION}

In this section, we look deeper at the patterns that emerged in the data.

\section{A. Overlap between Justification Categories}

As the Venn diagram in Fig. 1 shows, all 24 statements that were grouped into multiple categories had Change as one of the categories. The other code(s) given to these Justifications are, directly or indirectly, related to knowing the amount of that change. For example, Danny talks about holding a variable constant:

Danny [IQ4]: You look for variables that you can either set, so I give it a certain value, or control that it doesn't change... Those are the independent variables.

Emerson talks about Measurability and Change when using a calorimeter to measure change:

Emerson [Gas]: I'm going to use a calorimeter and heat it up and figure out how much heat has been absorbed to get the heat capacity, and therefore know for a given change of temperature how much heat I'm getting.

Avery talks about a Functional Relationship as a means for predicting a change in temperature:

Avery [300K]: I would not have enough information about the comet to make the prediction to set [temperature] as my independent variable. I would have to use the feedback from my laser to understand and project how the temperature is changing as a function of time [emphasis added]. After I do a short experiment to calibrate that, then I might be able to.

In each of these cases, experts are comfortable treating the changing variable as independent when the amount of change for that variable is known. The Control, Measurability, and Functional Relationship Justifications would then seem to be different ways of determining how a change is known. Taken together, 69 of our 82 statements correlate independently knowing (Control, Measurability, and/or Functional Relationship) what the value of a variable will be (Change) with the variable being independent (41, RQIV) or an indicator of the appropriate thermodynamic potential (28, RQTP).

\section{B. Expert Flexibility}

Experts are flexible problem solvers, as evident by the various ways they use Control, Measurability, and Functional Relationship in different situations. Individual experts, however, demonstrate different levels of flexibility for the same situations. For example, in the $300 \mathrm{~K}$ question, Danny asserts that temperature is not independent:

Danny [300K]: If you adjust your energy beam...you can aim [the temperature] at the value you want to have, but it's an indirect meas-it's a consequence of-it's a dependent variable. And you simply change the independent variable in such a way that the dependent variable is what you want. 
Danny rejects temperature as an independent variable because it is the energy of the laser that is directly controlled. MV follows up:

$\boldsymbol{M V}$ : So you [view] the thing you can "turn a knob on" as the independent variable?

Danny: Yeah, exactly. So, what can I control? I have no direct control over the temperature, I'm not connected to a heat bath or anything like that, but in this case, I can add energy.

Danny (possibly referring back to the Ice scenario) argues that control of an external heat bath constitutes controlling the temperature of the system, allowing temperature to be the independent variable. However, direct control over the external laser power is insufficient to treat temperature as independent in the $300 \mathrm{~K}$ scenario. Avery, however, would seem to disagree:

Avery [300K]: I would have to use the feedback from my laser to understand and project how the temperature is changing as a function of time.

As discussed in Section IV A, Avery does not initially know the Functional Relationship between laser power and comet temperature and is thus unwilling to treat temperature as independent. However, Avery appears to have no problem with temperature being independent if this relationship is found. Emerson makes a similar claim (also in Section IV A) for the heavier-than-air gas scenario by suggesting that finding the heat capacity of the gas would allow temperature to be the independent variable, despite Emerson's "knob" for the system being an energy, rather than temperature, "knob."

An area where our experts tended to show less flexibility, however, was in answering the general questions IQ1-IQ4. Answers to these questions relied almost exclusively on only Control and Change justifications (as shown in Table I), which may indicate that our experts were not employing all of their problem-solving resources (e.g. Measurability and Functional Relationship) to answer these questions.

\section{Choice}

Charlie is the only expert to explicitly state that any variables could be considered independent:

Charlie: We are free to think of whatever we like as independent variables, it's just sometimes more or less productive. And sometimes experts will make leaps...novices will not be able to make.
Other experts also discuss making a decision about which variables or thermodynamic potentials to use, though they do not state or suggest that any variable in the system could be considered independent:

Avery: When choosing what variables are relevant for your system, you need to look at what parameters you have control over.

Blair: [Legendre transformations] allow you to quickly choose between which state variables...you use to describe your system. You want to choose them in terms of what you try to control and what you want to look at.... What you want to measure.

Avery and Blair focus their attention on what factors they would consider while making decisions about independent variables. This behavior suggests that they may view this element of choice in the system as an aspect of the problem to be solved (which is interesting considering that, throughout the interview, the experts made different choices from one another). This approach also contrasts with Charlie's less constrained suggestion that the experimenter is free to select any variables as independent. The extremely low frequency of this less constrained notion of choice in our interviews surprised us and contrasts with the clear advice given in [4]: "Some concepts worth emphasizing include the freedom to choose and to use convenient independent variables...[and] thermodynamic potentials."

\section{CONCLUSIONS}

Despite differences in their approaches, the experts we interviewed were all able to navigate the proposed scenarios productively. They discussed change, control, measurement, and known relationships as possible considerations for determining independent variables. We conclude that experts understand that they have some freedom to pick their independent variables, that their choice of independent variables is informed by what they know about the way variables change, and that there are different ways to know the amount that variables change. Whether these considerations are effectively communicated to, and understood by, students is and area of further research.

\section{ACKNOWLEDGEMENTS}

We thank the experts who agreed to be interviewed as well as the Oregon State University PER Group. This research was supported in part by NSF DUE-1323800.
[1] R. K. Zia, E. F. Redish, and S. R. McKay, Am. J. Phys. 77, 614 (2009).

[2] M. Vignal, C. Manogue, D. Roundy, and E. Gire, in Physics Education Research Conference 2017, PER Conference (Cincinnati, OH, 2017) pp. 404-407.
[3] M. Miles, A. Huberman, and J. Saldaña, Qualitative Data Analysis (SAGE Publications, 2013).

[4] J. W. Cannon, American Journal of Physics 72, 753 (2004), https://doi.org/10.1119/1.1648327. 